\title{
La place des lipides dans les compléments nutritionnels oraux $(\mathrm{CNO})^{\text {it }}$
}

\author{
Cécile Bonhomme ${ }^{1,2, *}$ \\ ${ }^{1}$ Syndicat Français de la Nutrition Spécialisée, 75008 Paris, France \\ 2 Delical-Lactalis Nutrition Santé, France, 35370 Torcé, France
}

Reçu le 31 janvier 2019 - Accepté le 21 mars 2019

\begin{abstract}
Résumé - La dénutrition touche 2 millions de personnes en France et a pour conséquence une augmentation des complications, des infections et du risque de mortalité ; en découle une qualité de vie fortement impactée, des capacités fonctionnelles diminuées et un risque de dépendance. Les compléments nutritionnels oraux $(\mathrm{CNO})$, considérés comme des DADFMS ${ }^{1}$, sont une des premières étapes de la renutrition, et restent une solution physiologique et non invasive à la dénutrition protéino-énergétique. Les CNO hypercaloriques et hyperprotéinés comblent le manque d'apport alimentaire, sans augmenter grandement les volumes d'aliments consommés. La formulation des CNO est encadrée par le règlement délégué (UE) 2016/128 sur les DADFMS. La HAS ${ }^{2}$ préconise que les CNO doivent apporter au moins $30 \mathrm{~g}$ de protéines et/ou $400 \mathrm{kcal}$ par jour. A ce jour, de nombreux CNO sont proposés, afin de répondre aux besoins nutritionnels, aux textures adaptées et aux goûts des sujets dénutris. Selon la catégorie, les CNO contiennent des lipides, à hauteur de 26 à $42 \%$ de l'AET et d'origines variées : colza, tournesol, lipides laitiers. Or, dans la prise en charge de la dénutrition, tous les lipides ne sont pas équivalents ; s'ils fournissent tous l'énergie indispensable, certains seraient impliqués dans la modulation de l'inflammation, d'autres dans l'équilibre de la flore intestinale ou des fonctions cognitives. Ils peuvent ainsi contribuer à l'efficacité des $\mathrm{CNO}$ dont le rôle majeur est l'amélioration du statut nutritionnel des patients afin qu'ils puissent être soignés plus efficacement.
\end{abstract}

Mots clés : dénutrition protéino-energétique / compléments nutrionnels oraux (CNO) / Denrées Alimentaires Destinées à des Fins Médicales Spéciales (DADFMS) / lipides

\begin{abstract}
Lipids in oral nutritional supplements. Malnutrition affects 2 million people in France and results in an increase in risks of complications, infections and mortality; this results in a highly impacted quality of life, reduced functional capacities and a risk of dependence. Oral nutritional supplements (ONS), considered as FSMP ${ }^{3}$, are one of the first steps in re-nutrition, and remain a physiological and non-invasive solution to protein-energy malnutrition (PEM). High-calorie and high-protein ONS ill the food intake deficit, without greatly increasing the volumes of food consumed. The formulation of the ONS is governed by the delegated regulation (EU)2016/128 on FSMP. The French National Health Authority (HAS) recommends that ONS should provide at least $30 \mathrm{~g}$ of protein and/or $400 \mathrm{kcal}$ per day. To date, many ONS are available to meet the nutritional needs, adapted textures and tastes of malnourished patients. Depending on the category, ONS contain lipids accounting for 26 to $42 \%$ of the total energy intake and from various origins: rapeseed, sunflower, dairy lipids. However, in the management of malnutrition, not all lipids are equivalent; if they all provide essential energy, some would be involved in modulating inflammation, others in balancing the gut microbiota or cognitive
\end{abstract}

\footnotetext{
Contribution to the Topical Issue "Lipids in the elderly: needs, nutrition and physiopathology / Les lipides pour les seniors : besoins, alimentation et physiopathologie"

*Correspondance : cecile.bonhomme@lactalis.fr

${ }^{1}$ Denrées Alimentaires Destinées à des Fins Médicales Spéciales.

${ }^{2}$ Haute Autorité de Santé.

${ }^{3}$ Food for Special Medical Purposes.
} 
functions. They can thus contribute to the effectiveness of ONS whose major role is to improve the nutritional status of patients so that they can be treated more effectively.

Keywords: protein-energy malnutrition / Oral Nutritional Supplements (ONS) / Food for Special Medical Purposes (FSMP) / lipids

La dénutrition touche 2 millions de personnes en France (Clerc, 2017) et $10 \%$ de la population européenne (PNNS, 2010). Ce problème de santé publique majeur affecte toutes les populations, quel que soit l'âge et le milieu socio-culturel. En France, elle concerne de 8 à $20 \%$ des enfants hospitalisés (PNNS, 2010; Hankard et al., 2012) et la prévalence de la dénutrition protéino-énergétique augmente avec l'âge et varie selon les lieux de vie (hôpital, maison de retraite, domicile): elle affecte de 4 à $10 \%$ des personnes âgées vivant à domicile, pour s'élever à $15-38 \%$ chez les séniors en institution et à 30$70 \%$ chez les malades hospitalisés (HAS (Reco), 2007). Les patients atteints de pathologies telles que le cancer sont tout particulièrement à risque d'être dénutri. Ainsi la prévalence de la dénutrition chez les patients cancéreux est de 20 à $70 \%$ selon les études, avec des différences liées à l'âge du patient, au type de cancer et au stade du cancer (Arends et al., 2017). Pour traiter la dénutrition, les compléments nutritionnels oraux (CNO) sont fortement recommandés par les autorités et associations spécialisées, et sont légalement considérés comme des denrées alimentaires destinées à des fins médicales spéciales (DADFMS). Leur composition lipidique n'est pas réglementée mais mérite un intérêt particulier. En effet un certain nombre d'études interpelle sur les effets bénéfiques de certains lipides chez les patients dénutris.

\section{La dénutrition}

Selon l'Agence nationale de l'accréditation et de l'évaluation en santé (ANAES), la dénutrition protéino-énergétique se définit comme "la résultante d'un déséquilibre entre les apports et les besoins protéino-énergétiques de l'organisme. Ce déséquilibre entraîne des pertes tissulaires ayant des conséquences fonctionnelles délétères. Il s'agit d'une perte tissulaire involontaire» (Anaes, 2003). La 'European Society for Clinical Nutrition and Metabolism' (ESPEN) complète en précisant que la dénutrition se caractérise par des critères phénotypiques et étiologiques: (a) une perte de poids involontaire $>5 \%$ en 6 mois ou $>10 \%$ au-delà de 6 mois ; (b) un faible Indice de Masse Corporelle (IMC) : $<20 \mathrm{~kg} / \mathrm{m}^{2}$ pour les personnes âgées de moins de 70 ans et $<22 \mathrm{~kg} / \mathrm{m}^{2}$ pour celles de plus de 70 ans; (c) une réduction de la masse musculaire : évaluée par des techniques validées de mesure de la composition corporelle; (d) une réduction de la prise alimentaire et de l'assimilation: $\leq 50 \%$ des besoins énergétique pendant plus d'une semaine, ou toute réduction pour plus de 2 semaines, ou tout trouble gastro-intestinal chronique ayant un impact négatif sur l'assimilation ou l'absorption des aliments; (e) une inflammation (Cederholm et al., 2018).

Les causes physiologiques de la dénutrition protéinoénergétique sont multifactorielles et classées comme suit: (a) apport alimentaire altéré ; (b) besoins métaboliques altérés; (c) troubles de la digestion et/ou de l'absorption; et (d) pertes excessives d'éléments nutritifs (Marshall, 2018) (Tab. 1). Ces diverses causes sont souvent couplées: à titre d'exemple une personne âgée sarcopénique présentera une diminution de l'appétit (anorexie du vieillissement) ainsi qu'une moins bonne utilisation des nutriments, en adéquation avec un vieillissement normal (Ferry, 2010; Marshall, 2018). La perte excessive de nutriments peut survenir lors de dysfonctionnements gastrointestinaux (diarrhée, stéatorrhée, vomissements, etc.), mais également résulter de saignements internes ou gastro-intestinaux, de fistules, etc. (Marshall, 2018). Ces causes de dénutrition risquent fortement d'être liées à un stress métabolique chronique dû au vieillissement lui-même ou à des pathologies qui sollicitent fortement les ressources énergétiques de l'organisme. En effet, l'hypercatabolisme, à l'origine d'une augmentation des besoins, est déclenché lors de toute maladie infectieuse, d'une destruction tissulaire (infarctus, accident vasculaire cérébral, etc.) ou d'une réparation tissulaire lors de fracture ou d'escarres (Ferry, 2010; De Bandt, 2015). L'hypercatabolisme est particulièrement dangereux chez le sujet âgé car il survient sur un organisme qui présente déjà une diminution de ses réserves nutritionnelles et des déséquilibres métaboliques : trouble du métabolisme du glucose avec retard de sécrétion d'insuline et résistance périphérique à l'insuline entraînant une tendance à l'hyperglycémie après un repas ou lors d'un stress (maladie) ; trouble du métabolisme protéique avec préservation du catabolisme protéique mais diminution de l'anabolisme de 20 à $30 \%$ (Raynaud-Simon et Lesourd, 2000). Les personnes âgées présentent une résistance anabolique en répondant plus difficilement aux stimuli anaboliques normalement robustes tels que les acides aminés et les exercices de résistance (Breen et Phillips, 2011). L'insuline est la principale hormone anabolique postprandiale et la résistance à son action contribue à la sarcopénie en ne stimulant plus assez la synthèse protéique (Morais et al., 2018). La diminution des ingesta est également un facteur clef de la dénutrition: elle peut être liée aux traitements (chimiothérapie modifiant le goût des aliments, ou polymédication), à la pathologie elle-même (aversion pour les protéines chez l'insuffisant rénal hors séance de dialyse), à la douleur, aux capacités fonctionnelles (dentition, mastication, déglutition, capacité à se nourrir seul) ou cognitives. À ces éléments s'ajoutent les facteurs psycho-sociologiques comme l'isolement, le deuil, etc. (HAS (Synthèse), 2007; Marshall, 2018).

Les conséquences de la dénutrition sont importantes puisqu'elle est à l'origine d'une diminution des défenses de l'organisme (diminution du nombre de cellules immunitaires) (Ferry, 2010; Crichton et al., 2019) menant à un risque accru d'infection nosocomiale (Potter et al., 1995; Paillaud et al., 2005). En l'absence d'augmentation des apports alimentaires les nutriments sont prélevés sur les réserves de l'organisme: les acides aminés sont obtenus par la protéolyse musculaire, le calcium par la lyse osseuse, le glucose par stimulation de la néoglucogenèse et les acides gras par lyse adipocytaire (Ferry, 2010). Ainsi la dénutrition accélère la perte de masse musculaire, viscérale et osseuse, augmentant le risque de chutes et de fractures (Ferry, 2010; Crichton et al., 2019); elle augmente le risque de développer des escarres et empêche 
Tableau 1. Modifications de la prise alimentaire et du métabolisme chez les personnes âgées.

\begin{tabular}{|c|c|c|}
\hline $\begin{array}{l}\text { Types de modifications } \\
\text { Modifications de la prise alimentaire } \\
\text { (liées au vieillissement) }\end{array}$ & \multicolumn{2}{|c|}{ Effets } \\
\hline & $\begin{array}{l}\text { Diminution de l'appétit et de la sensation } \\
\text { de soif }\end{array}$ & $\begin{array}{l}\text { Avec sensation de satiété précoce et prolongée } \\
\text { Avec ralentissement de la digestion et } \\
\text { l'allongement de la période d'anorexie après le } \\
\text { repas }\end{array}$ \\
\hline & $\begin{array}{l}\text { Altération de la denture, des capacités de } \\
\text { mastication et diminution de la salivation }\end{array}$ & Contribue à la perte de l'appétit \\
\hline & $\begin{array}{l}\text { Moindre contrôle du métabolisme du } \\
\text { glucose }\end{array}$ & $\begin{array}{l}\text { Hyperglycémie d'après repas due à une relative } \\
\text { résistance à l'insuline }\end{array}$ \\
\hline & & Elle est aggravée par la résistance anabolique \\
\hline \multirow[t]{4}{*}{ Modifications métaboliques } & $\begin{array}{l}\text { Modifications du métabolisme du calcium } \\
\text { et de la vitamine D, fragilité osseuse } \\
\text { (ostéoporose) }\end{array}$ & $\begin{array}{l}\text { Diminution de la synthèse de la vitamine D. } \\
\text { Une supplémentation systématique est } \\
\text { recommandée }\end{array}$ \\
\hline & & $\begin{array}{l}\text { Capacités d'absorption digestive et rénale du } \\
\text { calcium alimentaire diminuées }\end{array}$ \\
\hline & Déséquilibre du métabolisme de & Altération de la fonction rénale \\
\hline & $\begin{array}{l}\text { l'eau-Augmentation du risque de } \\
\text { déshydratation }\end{array}$ & Altération de la sensation de soif \\
\hline
\end{tabular}

la cicatrisation (Taylor, 2017); la dénutrition peut étendre la durée du séjour hospitalier (Herrmann, 1992) et finalement augmenter les risques de mortalité (Marshall, 2018). La qualité de vie est aussi fortement impactée avec une diminution des capacités fonctionnelles et un risque de dépendance (Marshall, 2018).

\section{Les compléments nutritionnels oraux (CNO)}

Selon l'ESPEN, en cas de risque de dénutrition il est recommandé d'initier précocement une prise en charge nutritionnelle par des CNO et/ou une alimentation entérale par sonde (HAS (Arg), 2007). Dans ce cadre, les CNO représentent, après l'enrichissement de l'alimentation, la première étape de la re-nutrition, sans l'aide de sonde ou de cathéter; il s'agit de la technique de re-nutrition la plus physiologique et la moins agressive (Cessot et al., 2014). Les $\mathrm{CNO}$ ont pour vocation de répondre aux besoins des populations dénutries puisqu'ils se définissent comme des Denrées Alimentaires Destinées à des Fins Médicales Spéciales (DADFMS) (Arrêté du 2 déc., 2009). Ainsi, le règlement européen 2016-128 concernant les exigences relatives aux DADFMS s'applique (Règl. UE 2016/128, 2016). Les CNO font partie de la catégorie «aliments incomplets du point de vue nutritionnel, qui, avec une composition normale ou adaptée pour répondre aux besoins nutritionnels propres à une maladie, à un trouble ou à un état de santé, ne peuvent pas constituer la seule source d'alimentation » (Règl. UE 2016/128, 2016).

\subsection{Recommandation et composition des CNO}

Quelle que soit la cause de la dénutrition, les CNO apportent une solution au problème en concentrant dans un soin nutritionnel l'énergie et les protéines nécessaires pour combler le manque d'apport alimentaire, sans trop augmenter les volumes d'aliments consommés. En France, la Haute Autorité de Santé (HAS) recommande de prescrire des CNO qui apportent sur la journée environ $1 / 3$ des apports quotidiens et au moins $30 \mathrm{~g}$ de protéines et $400 \mathrm{kcal}$ (HAS (Reco), 2007). Il est recommandé de favoriser les produits hyperénergétiques ( $\geq 1,5 \mathrm{kcal} / \mathrm{ml} \mathrm{ou} / \mathrm{g}$ ) et/ou hyperprotidiques (protéines $\geq 7,0 \mathrm{~g}$ / $100 \mathrm{ml} \mathrm{ou} / 100 \mathrm{~g}$ ou protéines $\geq 20 \%$ des Apports Energétiques Totaux (AET)) (HAS (Reco), 2007). Le règlement 2016-128 précise les exigences portant sur la composition des DADFMS mais n'apporte aucun élément précis sur la composition lipidique des CNO à part qu'ils «présentent une composition basée sur de solides principes médicaux et nutritionnels. Leur utilisation, conformément aux instructions des fabricants, est sûre, adaptée et efficace pour répondre aux besoins nutritionnels particuliers des personnes auxquelles les denrées sont destinées, tels qu'établis par des données scientifiques généralement admises » (Règl. UE 2016/128, 2016). Aucune recommandation n'est émise sur la composition de l'apport 
lipidique dans la mesure où la notion de complémentation nutritionnelle implique le maintien d'un certain niveau d'alimentation orale spontanée. Il n'est donc pas indispensable que les valeurs en triglycérides à chaîne longue (TCL) ou moyenne (TCM), en acides gras polyinsaturés/mono-insaturés/saturés et en omégas-3 et -6 soient proches des apports nutritionnels conseillés (ANC) des sujets sains (Cessot et al., 2014).

\subsection{Les différents types de CNO}

Il existe une grande variété de $\mathrm{CNO}$, qui se différentient selon différents critères: (a) leurs compositions: densité énergétique et teneur en protéines (hyperccaloriques (HC) et/ ou hyperprotidiques (HP)), avec ou sans lactose, avec ou sans gluten, avec ou sans fibres, avec ou sans édulcorants ; (b) leurs présentations : poudre, crème, boisson, potage, etc.; (c) leurs conditionnements: à reconstituer, prêt à l'emploi, de type «manger-main », etc. ; (d) leurs textures : liquide, crème, semiliquide, semi-solide, solide, etc. ; (e) leurs goûts : sucré, salé ; (f) leurs arômes et saveurs, etc. (Cessot et al., 2014). Cet éventail de $\mathrm{CNO}$ permet de répondre aux besoins des patients, que ce soit sur le plan nutritionnel, celui du goût et de l'appétence, ou en relation avec un éventuel handicap (troubles de déglutition, difficultés de préhension des objets, etc.) (HAS (Reco), 2007).

Ainsi, il existe des produits sucrés, salés ou natures se présentant sous différentes textures : liquides (boisson lactée, boisson fruitée, potage), semi-liquides (brassé, riz au lait), semi-solides (crème dessert, dessert aux fruits...), et solides (biscuit), avec, pour chaque catégorie, différents arômes et saveurs (HAS (Reco), 2007). Il existe aussi des poudres de protéines ou de maltodextrines. La composition lipidique des produits dépend de la catégorie de CNO. Une observation du marché montre que les $\mathrm{CNO}$ sans lactose contiennent essentiellement des huiles de colza et tournesol ou de l'huile de colza seule. Les CNO lactés contiennent des lipides laitiers, associés à l'huile de colza et tournesol. Certains contiennent des triglycérides à chaînes moyennes (TCM). La majorité contient des lipides à hauteur de 26 à $42 \%$ de l'AET sauf les boissons fruitées qui sont dépourvues de lipides. Le rapport acide linoléique/acide alpha-linolénique est majoritairement inférieur à 5. Enfin, rares sont les produits contenant des huiles de poisson.

\subsection{Les preuves d'efficacité des CNO}

Des études menées chez des personnes âgées hospitalisées et dénutries ont montré une amélioration du poids et de la survie, ainsi qu'une réduction de la survenue de complications après une prise en charge nutritionnelle orale (HAS (Arg), 2007) (Gammack et Sanford, 2015). Plus spécifiquement la méta-analyse de Milne et al. (2009) incluant 62 études et plus de 10000 personnes âgées à risque de dénutrition, a montré qu'une prise de $\mathrm{CNO} \mathrm{HP} / \mathrm{HC}$ augmente de façon significative le poids de 2,2\% (Milne et al., 2009). Ces résultats positifs sont également observés chez des personnes âgées atteintes de démence (Allen et al., 2013) et une majorité d'individus atteints de cachexie cancéreuse (selon le type de cancer et de traitement) (Kim et Sung, 2016; Laviano et al., 2016). Une autre revue de la littérature (Cawood et al., 2012) montre que la prise en charge de la dénutrition par des $\mathrm{CNO}$ hyperprotidiques permet d'augmenter significativement le poids et la force musculaire de préhension, et de réduire significativement les complications $(-30 \%)$ et les réadmissions à l'hôpital $(-40 \%)$. Enfin une étude récente observationnelle prospective française, sur des patients âgés dénutris vivant à domicile, montre une bonne observance du traitement à 1 mois $(83,5 \%)$, avec une augmentation de l'appétit supérieure à 6 mois dans le groupe ayant reçu des CNO ainsi qu'une réduction des coûts de santé et du risque d'hospitalisation dans le groupe ayant reçu plus de $500 \mathrm{kcal}$ ou $30 \mathrm{~g}$ de protéines.

L'efficacité des CNO a souvent été mise à mal par l'idée que la supplémentation calorique réduit l'apport calorique des repas. Cela a été maintes fois réfuté puisque lorsque des suppléments sont fournis entre les repas, la consommation d'aliments provenant des plateaux-repas ne diminue pas (Gammack et Sanford, 2015).

\section{L'intérêt des lipides dans les CNO}

\subsection{Le statut lipidique altéré des individus dénutris}

Dans les cas de dénutrition, le statut lipidique est altéré. Ainsi, d'après Omran et Morley (2000) les taux de cholestérol sériques inférieurs à $160 \mathrm{mg} / \mathrm{dL}$, ce qui correspond à une hypocholestérolémie, seraient le reflet de la dénutrition (Omran et Morley, 2000). Cette observation est confirmée par une étude clinique réalisée sur des sujets âgés dénutris hospitalisés dans une unité de soins médicaux gériatriques dont les teneurs en cholestérol total, HDL cholestérol et apoprotéines A et B sont inférieures à la population normale (Monarque-Favard et al., 2002). Une récente méta-analyse confirme que le cholestérol total est statistiquement plus bas chez les personnes âgées à risque de dénutrition et peut être considéré comme un des marqueurs de dénutrition (Zhang et al., 2017).

Ces altérations affectent les teneurs en acides gras. Chez des rats âgés, la dénutrition diminue les taux sériques d'acide alphalinolénique (ALA) ainsi que ceux des acides gras omega-3 à chaîne longue: acides eicosapentaénoique (EPA) et docosahexaénoique (DHA). Il est également noté une légère augmentation des acides gras $n-6$ : acides linoléique (LA) et arachidonique (AA). Cela résulte en un ratio AA/EPA augmenté et une stimulation de l'inflammation (Delplanque, 2014).

Si le statut des lipides sanguins des individus dénutris est altéré, la perte de poids engendre une réduction importante des réserves lipidiques. Dans l'organisme, le tissu adipeux blanc a pour fonction première le stockage de l'énergie, sous forme de triglycérides (Vaitkus et Celi, 2017). La dégradation du tissu adipeux, i.e. la lipolyse, est certainement l'élément le plus évident de la contribution de ce tissu à la dénutrition (Vaitkus et Celi, 2017). Dans le cas du cancer, ce phénomène est la réponse à un bilan énergétique négatif dû à une anorexie associée au cancer et à d'autres facteurs pathologiques (Vaitkus et Celi, 2017). Il est également postulé que les acides gras libérés par la lipolyse peuvent servir de substrats à l'oxydation (Bing, 2011) et permettre de faire face à une augmentation de la demande énergétique; en cas de carence glucidique à long terme la lipolyse permettrait de prolonger le temps de survie en épargnant la masse protéique. En effet, l'expression génique de CIDEA (cell death-inducing DNA 
fragmentation factor-a-like effector $A$ ) dans le tissu adipeux est augmentée chez les patients atteints de cachexie cancéreuse et sa surexpression dans les adipocytes stimule l'oxydation des acides gras tout en diminuant l'oxydation du glucose, probablement par inactivation du complexe pyruvate déshydrogénase (Bing, 2011).

Une réduction importante des réserves lipidiques induit, chez les personnes dénutries, une perte de chance face à l'agression qui nécessite toutes les ressources énergétiques de l'organisme.

\subsection{Le rôle et la qualité des lipides dans le traitement de la dénutrition}

\subsubsection{Les lipides comme source d'énergie}

Pour éviter la fonte de masse musculaire et graisseuse chez les individus dénutris il est important d'augmenter l'apport énergétique. La HAS recommande de prescrire des CNO permettant d'atteindre un apport alimentaire supplémentaire d'au moins $400 \mathrm{kcal} / \mathrm{j}$ (Assurance Maladie, 2015). Il est recommandé de pratiquer une activité physique et d'augmenter la densité énergétique du bol alimentaire en maintenant son volume constant avec une alimentation enrichie (Hébrant, 2014). Or, les lipides ont une valeur énergétique par gramme supérieure $(9 \mathrm{kcal})$ à celle des protéines et des glucides $(4 \mathrm{kcal})$ et constituent un excellent moyen d'augmenter l'apport énergétique dans un petit volume (Schneyder, 2014).

D'après Hébrant (2014) différents traitements de la cachexie sont à l'essai dont la supplémentation en acides gras polyinsaturés (AGPI ou PUFA pour PolyUnsaturated Fatty Acids) oméga-3 qui pourrait diminuer le catabolisme des protéines et l'anorexie, et maximiser la réponse à la chimiothérapie (Hébrant, 2014). Chez les malades cachectiques porteurs d'un cancer, une complémentation en AGPI n-3 d'au moins $2 \mathrm{~g} / \mathrm{j}$ ralentit la perte pondérale (Hébuterne, 2012).

\subsubsection{Rôle des lipides dans la régulation de la masse et fonction musculaire}

Plusieurs études in vitro et in vivo suggèrent que les acides gras saturés et insaturés peuvent agir pour réguler de manière différentielle la masse et la fonction du muscle squelettique (Lipina et Hundal, 2017; Tachtsis et al., 2018) :

- par exemple, une étude in vitro a démontré que l'exposition des myotubes $\mathrm{C} 2 \mathrm{C} 12$ (cellules fusionnées qui se différencient en fibres musculaires) au palmitate (C16:0) diminuait le diamètre du myotube et supprimait la signalisation de l'insuline (Bryner et al., 2012). En revanche, l'application du DHA ne modifie pas la morphologie du myotube lorsqu'il est appliqué seul et permet de lutter contre l'atrophie induite par le palmitate dans les myotubes C2C12 (Bryner et al., 2012);

- il a été démontré que des souris ayant développé l'adénocarcinome du colon-26, un modèle animal de cachexie cancéreuse, recevant un régime alimentaire enrichi en acide linoléique conjugué, préservait la masse musculaire gastrique. Notamment, cet effet protecteur coïncide avec une réduction de l'expression du récepteur $\mathrm{du}$ TNF- $\alpha$ (tumor necrosis factor) au niveau du muscle squelettique, ce qui suggère que les AGPI pourraient empêcher, au moins en partie, la fonte musculaire, en réduisant les actions cataboliques de la cytokine TNF- $\alpha$ (Graves et al., 2005; Langen et al., 2006);

- une supplémentation alimentaire en EPA a atténué la dégradation des protéines dans le muscle gastrocnémien de souris atteintes de la tumeur MAC16 induisant la cachexie (Whitehouse et al., 2001);

- de plus, les hamsters dystrophiques nourris avec un régime enrichi en acide $\alpha$-linolénique (ALA) présentent une amélioration de la morphologie et de la fonction musculaire (Fiaccavento et al., 2010);

Ensemble, ces études in vitro et in vivo appuient la notion selon laquelle certains acides gras offrent une protection contre la fonte musculaire en réponse à diverses conditions pathologiques (Lipina et Hundal, 2017). Des analyses se concentrant sur des patients âgés en bonne santé confirment ces résultats et suggèrent que les AGPI oméga-3 stimulent la synthèse des protéines musculaires induite par l'hyperaminoacidémie et l'hyperinsulinémie, neutralisant ainsi la résistance anabolique et la sarcopénie (Smith et al., 2011; Di Girolamo et al., 2014).

\subsubsection{La modulation de l'inflammation}

L'inflammation systémique est un élément aggravant de la dénutrition et de la perte de masse musculaire. Dans le cas d'une maladie sous-jacente telle que le cancer, des cytokines proinflammatoires (TNF- $\alpha$ et interleukine-6) sont produites en excès par la tumeur provoquant une inflammation systémique, perturbant le métabolisme des glucides, des lipides et des protéines dans l'ensemble du corps et affectant le contrôle neuroendocrinien de l'appétit (Arends et al., 2017). Cela provoque donc une anorexie et une dégradation des tissus pouvant, à leur tour, entraîner une perte de poids importante, des modifications de la composition corporelle et une dégradation de la fonction physique (Arends et al., 2017). Chez les séniors, cette inflammation est tout aussi néfaste puisque l'organisme répond à toute infection et agression sévère par un syndrome d'hypercatabolisme. Or, ce dernier est particulièrement dangereux chez le sujet âgé qui présente déjà une diminution de ses réserves nutritionnelles et des déséquilibres métaboliques (RaynaudSimon et Lesourd, 2000).

Le rôle des omega-3, notamment EPA et DHA, dans l'inflammation est toujours controversé, mais il existe de fortes présomptions qu'un apport alimentaire de certains lipides peut moduler l'inflammation et agir de façon bénéfique sur la dénutrition:

- dans un modèle de rats âgés dénutris, un régime riche en matière grasse laitière, colza et DHA permet une meilleure restauration et amélioration du statut oméga-3 qu'un régime enrichi en huile de soja, ainsi qu'un meilleur statut inflammatoire. En effet, une dénutrition non carencée en acide alpha-linolénique (ALA) chez le rat âgé, induit une perte spécifique et conséquente des omega-3 (ALA, EPA et DHA). Un régime de renutrition contenant de l'huile de soja restaure les acides gras de la série n- 6 et n-3 à l'exception du DHA. La situation pro-inflammatoire, i.e. le ratio AA/EPA, est également restauré. Le régime riche en matière grasse laitière, colza et DHA restaure, voir même 
augmente les taux d'oméga-3, dont le DHA et réduit les taux les acides gras de la série n-6. Le ratio AA/EPA étant fortement diminué, la situation inflammatoire est améliorée (Delplanque, 2014);

- la synthèse des études d'intervention clinique avec de l'EPA sur le statut nutritionnel de patients atteints de cachexie cancéreuse, réalisée par Pappalardo et al. (2015) montre que l'EPA peut réduire l'inflammation et a le potentiel de moduler l'état nutritionnel et la composition corporelle (Pappalardo et al., 2015);

- d'autres sources de lipides ont été étudiées pour leur capacité à moduler l'inflammation : l'acide stéaridonique (18:4 n-3, SDA) est une source d'AGPI n-3 qui subit facilement une élongation pour devenir de l'ETA $(8,11,14,17$-eicosatetraenoic acid, 20:4 n-3) un isomère de l'acide arachidonique. L'étude in vitro de Gagnon et al. a démontré récemment que la SDA est un précurseur de métabolites possédant des caractéristiques anti-inflammatoires (Gagnon et al., 2018);

Un certain nombre de mécanismes d'action lié à l'apport alimentaire d'AGPI n-3, a été proposé : la quantité d'AA, connu pour être une source de prostaglandines E2 et de leucotriènes de la série 4 pro-inflammatoires, se retrouve limitée (Hébuterne, 2012; O’Connor et al., 2014); à l'inverse les AGPI n-3 qui favorisent la synthèse de prostaglandines de la série 3 , de leucotriènes de la série 5 et de médiateurs de la résolution de l'inflammation (comme les résolvines) sont accrus et associés à une amélioration de l'immunité via une réduction de la réponse inflammatoire (Calder, 2015).

Malgré ces exemples positifs, l'ESPEN considère, à ce jour, qu'il n'y a pas encore assez de résultats concluants pour reconnaître un rôle aux acides gras omega-3 sur la dénutrition (Arends et al., 2017).

Les omega-3 ne sont pas les seuls acides gras à présenter des effets bénéfiques : en 2006, l'étude de Dabadie et al. (2006) a évalué chez des moines, les effets d'apports modérés d'acide myristique (MA) laitier, à 1,2\% de l'énergie totale, associés à un apport en acide $\alpha$-linolénique de $0,9 \%$ sur les profils lipidiques et la fluidité des membranes des globules rouges. Les résultats montrent des profils lipidiques favorables et une augmentation de la fluidité membranaire des cellules en particulier chez les sujets les plus âgés (Dabadie et al., 2006). Cette fluidité membranaire, notamment dans les cellules immunitaires, est un mécanisme majeur de la modulation de l'inflammation, notamment via la transduction de signaux et l'expression de gènes (Calder, 2012).

\subsubsection{La présence de lipides alimentaires favoriserait l'observance}

Chez les malades hospitalisés, les CNO permettent un gain de poids et réduisent la morbidité et la mortalité. Cependant, l'observance des CNO est le principal facteur limitant leur efficacité (Galmiche et al., 2014). Une mauvaise observance est liée pour $43 \%$ à la lassitude et à la non-adéquation aux goûts du patient de la texture ou saveur proposée; vient ensuite le volume, et la difficulté à s'approvisionner à domicile.

Une des façons d'améliorer cette observance, est d'apporter des lipides. En effet, l'Homme présente une préférence spontanée pour les lipides alimentaires (Gaillard et al., 2008). Non seulement il est reconnu que les aliments riches en énergie sont attrayants et donc consommés de préférence (Besnard et al., 2016), mais les lipides jouent également un rôle majeur dans l'appétence via leurs propriétés texturales, olfactives et gustatives (Martin et al., 2010; Besnard et al., 2016). Les matières grasses participent aux attributs de texture et sont ainsi associées aux sensations de tendreté, moelleux, crémeux, onctueux, rondeur en bouche, fondant des aliments dont elles font partie (Souliac et al., 2010). D'autre part, la découverte récente de récepteurs lipidiques dans les papilles gustatives suggère que les lipides sont également détectés par la voie gustative, menant à l'idée d'un sixième sens gustatif consacré à la perception des lipides (Martin et al., 2010 ; Besnard et al., 2016). Ce sixième sens serait étroitement lié à la valeur de récompense hédonique des aliments, ce qui augmente la possibilité que le goût soit une force motrice importante menant à une consommation préférentielle d'aliments très savoureux, souvent riches en gras (Besnard et al., 2016). À noter que si les graisses alimentaires sont très majoritairement composées de triglycérides, ce sont les acides gras à longue chaîne (AGLC, nombre de carbones $>16$ ) qui sont responsables de l'attirance spontanée pour les lipides (Martin et al., 2010). Les lipides ont également pour intérêt de masquer le goût des protéines et des minéraux et de lubrifier le bol alimentaire pour une meilleure déglutition. En effet, en s'étalant sur la langue, les matières grasses agissent comme lubrifiant (Souliac et al., 2010).

Si ces éléments sont en faveur de l'apport de lipides dans les $\mathrm{CNO}$, certains AGPI oméga-3 peuvent être liés à des problèmes de stabilité à l'oxydation et de goût désagréable, comme cela a été décrit pour les suppléments alimentaires à base de lipides destinés aux enfants malnutris (Gaur et al., 2017). Cependant des études portant sur l'acceptabilité de CNO sont relativement positives: l'étude de García Almeida et al. (2017) porte sur l'acceptabilité, la conformité et la tolérance d'un supplément hyperprotéique riche en calories et enrichi en oméga-3, chez les patients cancéreux et démontre un taux d'observance de $100 \%$. $\mathrm{Ce}$ CNO spécifique était associé à un meilleur état nutritionnel des patients et inversait positivement la courbe de poids (García Almeida et al., 2017). De même, l'étude de Trabal Vilchez (2010) réalisée sur des patients atteints de cancer qui ont reçu un CNO enrichi en EPA, montre que tous les patients du groupe expérimental remplissaient les exigences minimales en matière d'observance de la supplémentation avec une dose moyenne de 1,6 g/j d'EPA (Trabal Vilchez, 2010).

\section{Les perspectives}

Les lipides ont définitivement un rôle à jouer dans les CNO. S'il existe toujours des désaccords concernant leur capacité à moduler l'inflammation ou à réguler la dénutrition, des recherches sont également nécessaires concernant la lipotoxicité et l'impact sur la dysbiose et le cognitif.

\subsection{Lipides et risque de lipotoxicité chez les personnes dénutries}

Il est reconnu que chez les personnes malnutries, notamment les individus obèses, un phénomène d'inhibition de l'effet anti-lipolytique de l'insuline se met en place et mène 
à une élévation chronique des acides gras dans la circulation sanguine, pouvant atteindre des niveaux toxiques dans les tissus non adipeux. Les effets délétères de l'accumulation de lipides dans les tissus non adipeux sont connus sous le nom lipotoxicité (Engin, 2017). Or, un phénomène similaire est suspecté chez les individus atteints de cachexie liée au cancer : la dégradation du tissu adipeux en réponse à un bilan énergétique négatif dû à une anorexie associée au cancer et à d'autres facteurs pathologiques est supportée par la réaction de lipolyse ; cette dernière serait initiée avant la perte musculaire et en serait responsable. La lipolyse entraîne une augmentation des acides gras libres dans la circulation, qui sont ensuite absorbés par le muscle squelettique. L'excès d'acides gras mènerait à l'accumulation de gouttelettes lipidiques dans les cellules du muscle squelettique provoquant des changements biochimiques (expression des gènes de l'ubiquitin lipases Atrogin-1 et de MuRF67) responsables de l'atrophie du muscle squelettique (Vaitkus et Celi, 2017). A ce jour il n'existe pas d'étude sur un éventuel effet lipotoxique des lipides alimentaires chez les individus dénutris; cependant il a été démontré que le cholestérol associé à des composés lipidiques alimentaires contribuaient au développement de la lipotoxicité chez la souris, notamment au niveau hépatique ; c'est le cas des AGPI n-6 dérivés de l'huile de soja (Henkel et al., 2018). Des recherches plus approfondies seront nécessaires pour comprendre les éventuels effets lipotoxiques de certains lipides alimentaires chez les individus dénutris.

\subsection{Dysbiose des personnes dénutries et supplémentation lipidique}

La modification de la composition et de la diversité du microbiote, phénomène connu sous le nom de dysbiose, est un facteur commun à l'obésité et la dénutrition, que cette dernière soit liée à une pathologie ou au vieillissement (de Clerq et al., 2016). Ainsi la dénutrition semble être associée à une réduction de la diversité et de la richesse bactérienne (Fluitman et al., 2017), elle-même associée à une modification du poids corporel et du stockage des graisses (de Clerq et al., 2016). Des études in vivo ont montré que le microbiote de souris néonatales sous-alimentées présentait une richesse et une diversité bactériennes globales plus faibles que celui des souris témoins bien nourries (Preidis et al., 2015).

Alors que la composition microbienne est largement étudiée dans les cas d'obésité et de résistance à l'insuline, les études sur le microbiote des personnes dénutris sont relativement rares (Fluitman et al., 2017). Cependant, il est noté qu'un microbiome associé à la dénutrition peut réduire la capacité de récolte et d'utilisation des nutriments. Il peut en outre avoir un effet satiétogène plus fort (Fluitman et al., 2017). Chez les patients dénutris souffrant d'anorexie mentale la dysbiose est fortement corrélée à la gravité de la dénutrition et à d'autres troubles métaboliques, ainsi qu'à une perturbation de la fonction intestinale (Hanachi et al., 2013, 2017).

L'étude de Yamamoto et al. (2018) a montré, in vivo, qu'un régime alimentaire enrichi en huile de poisson pourrait avoir des effets bénéfiques pour les personnes âgés dont la capacité d'absorption des lipides est diminuée (Yamamoto et al., 2018). En effet, l'étude sur des souris âgées montre que la diminution bactérienne liée à l'âge peut être retardée par un régime modérément gras enrichi en huile de poisson (Yamamoto et al.,
2018). Ce régime inhiberait également l'accumulation de lipides, notamment de cholestérol (Yamamoto et al., 2018).

\subsection{L'impact des lipides sur le cognitif}

L'avancée dans l'âge s'accompagne inexorablement d'un déclin cognitif considéré comme «normal». Ce dernier s'échelonne d'un simple dysfonctionnement cognitif dû à l'âge au développement de la démence (Heckman et al., 2017). Il est reconnu que les séniors atteints de démence risquent fortement de perdre du poids, de la masse musculaire et des capacités fonctionnelles. Par conséquent, l'utilisation des CNO a fait l'objet de nombreuses études. Une méta-analyse comprenant 12 études et incluant plus de 1000 personnes âgées institutionnalisées atteintes de démence montre une amélioration du poids de $3,5 \mathrm{~kg}$ chez les personnes recevant un CNO (avec un apport moyen de 390 calories supplémentaires par jour), ainsi qu'une amélioration significative des capacités cognitives (Allen et al., 2013).

Dans le cadre spécifique de la dénutrition, il n'existe, à notre connaissance, qu'une étude sur l'impact des lipides sur le cognitif: le modèle de rats âgés dénutris proposé par OuldHamouda, démontre que la dénutrition diminue les teneurs en AGPI oméga-3 dans le cerveau. Le ratio AA/AGPI oméga-3 est fortement augmenté, démontrant une situation proinflammatoire. Lors de la renutrition avec un régime riche en matière grasse laitière, colza, et DHA, les teneurs cérébrales en AGPI oméga-3 sont restaurées (Ould-Hamouda, 2015). Cette étude est d'autant plus intéressante que les acides gras oméga-3 jouent un rôle indispensable dans la construction, le maintien et le fonctionnement du cerveau (Ould-Hamouda, 2015). Cependant, à ce jour, les travaux menés sur l'effet des omega-3 sur le cognitif sont contradictoires. Les connaissances accumulées tendent à montrer que les omega-3 serait bénéfiques à des catégories de personnes âgées: les seniors en bonne santé, les personnes âgées souffrant de troubles de la mémoire ou de troubles cognitifs légers, les patients atteints de la maladie d'Alzheimer légère ou modérée (Cederholm, 2017 ; Canhada et al., 2018).

\section{Conclusion}

Dans les cas de dénutrition, qu'elle soit liée à l'âge ou à une pathologie, l'apport calorique provenant de la consommation d'aliments traditionnels est insuffisante. Une complémentation nutritionnelle orale (CNO) est nécessaire pour prévenir une perte de poids supplémentaire et favoriser la prise de poids (Gammack et Sanford, 2015). À ce jour une grande variété de CNO peut être proposée aux patients en fonction de leurs besoins, de leur goût et de leurs éventuels handicaps. Dans ce contexte, les lipides ont un rôle important à jouer, en permettant une concentration énergétique dans un volume restreint, en préservant le muscle, et rendant appétant les CNO, en améliorant la lubrification du bol alimentaire chez les patients ayant des troubles de la déglutition ou une déglutition douloureuse. Certains lipides pourraient présenter un intérêt santé pour les personnes dénutries : modulation de l'inflammation, amélioration de flore intestinale et des fonctions cognitives, etc. Cependant, les preuves sont encore insuffisantes et les limites sont importantes. Les CNO ne représentent 
qu'une part de l'alimentation. Ils doivent avant tout remplir le rôle majeur d'améliorer le statut nutritionnel des patients afin qu'ils puissent être soignés plus efficacement.

Remerciements. Le GLN d'avoir permis la présentation de ce sujet lors de la journée GLN du 6 novembre, Carine Dion (Foodinnov) pour sa participation à l'article.

Conflits d'intérêts. L'auteure est salariée de Lactalis Nutrition Santé

\section{Références}

Allen V, Methven L, Gosney M. 2013. Use of nutritional complete supplements in older adults with dementia: Systematic review and meta-analysis of clinical outcomes. Clin Nutr 32: 950-957.

ANAES. 2003. Rapport sur l'Évaluation diagnostique de la dénutrition protéino-énergétique des adultes hospitalisés. Service des recommandations professionnelles, pp. 1-15. Disponible sur https://www.has-sante.fr/portail/jcms/c_432199/fr/evaluationdiagnostique-de-la-denutrition-proteino-energetique-des-adulteshospitalises.

Arends J, Baracos V, Bertz H, et al. 2017. ESPEN expert group recommendations for action against cancer-related malnutrition. Clin Nutr 36: 1187-1196.

Arrêté du 2 déc. 2009. Arrêté du 2 décembre 2009 relatif à la modification de la procédure d'inscription et des conditions de prise en charge des nutriments pour supplémentation orale inscrits à la sous-section 1 , section 5 , chapitre $1^{\mathrm{er}}$, titre $\mathrm{I}^{\mathrm{er}}$, de la liste prévue à l'article L. 165-1 (LPP) du code de la sécurité sociale. $J$ Off Répub Fr 0284(22): 21182-21189.

Assurance Maladie. 2015. Dénutrition chez la personne âgée ( $>70$ ans) et aide à la prescription des compléments nutritionnels oraux (CNO). Ameli.fr, Mémos et fiches d'aide à la pratique, pp. 1-2. Disponible sur https://www.ameli.fr/llle-et-vilaine/medecin/ exercice-liberal/memos/prise-en-charge/complements-nutrition nels-oraux-cno.

Besnard P, Passilly-Degrace P, Khan NA. 2016. Taste of fat: A sixth taste modality? Physiol Rev 96: 151-176.

Bing C. 2011. Lipid mobilization in cachexia: Mechanisms and mediators. Curr Opin Support Palliat Care 5: 356-360.

Breen L, Phillips SM. 2011. Skeletal muscle protein metabolism in the elderly: Interventions to counteract the "anabolic resistance" of ageing. Nutr Metab 8: 68-71.

Bryner RW, Woodworth-Hobbs ME, Williamson DL, Alway SE. 2012. Docosahexaenoic acid protects muscle cells from palmitate-induced atrophy. ISRN Obes. 2012, 1-14.

Calder PC. 2012. Long-chain fatty acids and inflammation. Proc Nutr Soc 71: 284-289.

Calder PC. 2015. Marine omega-3 fatty acids and inflammatory processes: Effects, mechanisms and clinical relevance. Biochim Biophys Acta 1851: 469-484.

Canhada S, Castro K, Perry IS, Luft VC. 2018. Omega-3 fatty acids' supplementation in Alzheimer's disease: A systematic review. Nutr Neurosci 21: 529-538.

Cawood AL, Elia M, Stratton RJ. 2012. Systematic review and metaanalysis of the effects of high protein oral nutritional supplements. Ageing Res Rev 11(2): 278-296.

Cederholm T. 2017. Fish consumption and omega-3 fatty acid supplementation for prevention or treatment of cognitive decline, dementia or Alzheimer's disease in older adults - Any news? Curr Opin Clin Nutr Metab Care 20: 104-109.
Cederholm T, Jensen GL, Correia MITD, et al. 2018. GLIM criteria for the diagnosis of malnutrition-A consensus report from the global clinical nutrition community. Clin Nutr 3: pii: S0261-5614 (18)31344-X.

Cessot F, Desport J-C, Sautereau D. 2014. Complémentation nutritionnelle orale. HEGEL 4(3): S47-S49.

Clerc C. 2017. 2 millions de français dénutris. Pharma Janvier: 1-2.

de Clercq NC, Groen AK, Romijn JA, Nieuwdorp M. 2016. Gut microbiota in obesity and undernutrition. Adv Nutr Int Rev J 7: 1080-1089.

Crichton M, Craven D, Mackay H, Marx W, de van der Schueren M, Marshall S. 2019. A systematic review, meta-analysis and meta regression of the prevalence of protein-energy malnutrition: Associations with geographical region and sex. Age Ageing 48(1): 38-48.

Dabadie H, Motta C, Peuchant E, LeRuyet P, Mendy F. 2006. Variations in daily intakes of myristic and $\alpha$-linolenic acids in sn2 position modify lipid profile and red blood cell membrane fluidity. Br J Nutr 96: 283-289.

De Bandt J-P. 2015. Comprendre la physiopathologie de la dénutrition pour mieux la traiter. Ann Pharm Fr 73: 332-335.

Delplanque B. 2014. Impact of Denutrition and lipid quality for renutrition on plasma fatty acids and inflammatory markers in old rats. Stockholm, Sweden: ISSFAL International Congress, June 28-1 July.

Di Girolamo FG, Situlin R, Mazzucco S, Valentini R, Toigo G, Biolo G. 2014. Omega-3 fatty acids and protein metabolism: Enhancement of anabolic interventions for sarcopenia. Curr Opin Clin Nutr Metab Care 17: 145-150.

Engin AB. 2017. What is lipotoxicity? Adv Exp Med Biol 960: 197-220.

Ferry M. 2010. Nutrition, vieillissement et santé. Gérontol Soc. 33 (134): 123-132.

Fiaccavento R, Carotenuto F, Vecchini A, et al. 2010. An omega-3 fatty acid-enriched diet prevents skeletal muscle lesions in a hamster model of dystrophy. Am J Pathol 177: 2176-2184.

Fluitman KS, De Clercq NC, Keijser BJF, Visser M, Nieuwdorp M, IJzerman RG. 2017. The intestinal microbiota, energy balance, and malnutrition: Emphasis on the role of short-chain fatty acids. Expert Rev Endocrinol Metab 12: 215-226.

Gagnon KJ, Lefort N, Poirier SJ, Barnett DA, Surette ME. 2018. 5lipoxygenase-dependent biosynthesis of novel 20:4 n-3 metabolites with anti-inflammatory activity. Prostaglandins Leukot Essent Fat Acids 138: 38-44.

Gaillard D, Martin C, Passilly-Degrace P, Besnard P. 2008. Rôle des lipides dans la régulation du comportement alimentaire. Ol Corps Gras Lipides 15: 275-278.

Galmiche M, Alessandrini E, Eyrault E, et al. 2014. Influence de la concentration des compléments nutritionnels oraux sur leur observance et les apports protéino-énergétiques des patients hospitalisés: résultats d'une étude randomisée en cluster. JFHOD/ SNFGE 32-33.

Gammack JK, Sanford AM. 2015. Caloric supplements for the elderly. Curr Opin Clin Nutr Metab Care 18: 32-36.

García Almeida JM, Lupiáñez Pérez Y, Blanco Naveira M, et al. 2017. Adherencia y tolerancia como claves en la detención de la pérdida de peso en pacientes oncológicos sometidos a radioterapia mediante una estrategia de suplementación precoz con una fórmula enteral hipercalórica e hiperproteica específica. Nutr Hosp 34: 524-531.

Gaur S, Sloffer EM, Ojha A, et al. 2017. Omega-3-fortified lipidbased nutrient supplement: Development, characterization, and consumer acceptability. Food Nutr Bull 38: 158-171. 
Graves E, Hitt A, Pariza MW, Cook ME, McCarthy DO. 2005. Conjugated linoleic acid preserves gastrocnemius muscle mass in mice bearing the colon-26 adenocarcinoma. Res Nurs Health 28: $48-55$.

Hanachi MG, Levenez F, Latour E, et al. 2013. P081 Étude pilote du profil électrophorétique de diversité du microbiote intestinal chez les patients dénutris atteints d'anorexie mentale (AM). Nutr Clin Métab 27: S97.

Hanachi M, Manichanh C, Latour E, et al. 2017. Une dysbiose du microbiote intestinal comme facteur explicatif des troubles fonctionnels digestifs observés chez les patients dénutris sévères atteints d'anorexie mentale sous assistance nutritionnelle par voie entérale. Nutr Clin Métab 31, 232.

Hankard R, Colomb V, Piloquet H, et al. 2012. Dépister la dénutrition de l'enfant en pratique courante. Arch Pédiatr 19: 1110-1117.

HAS (Arg). 2007. Stratégie de prise en charge en cas de dénutrition protéino-énergétique chez la personne âgée-Argumentaire. Méd Mal. Métaboliques 1: 92-96.

HAS (Reco). 2007. Stratégie de prise en charge en cas de dénutrition protéino-énergétique chez la personne âgée-Recommandations. Méd Mal. Métaboliques 1: 92-96.

HAS (Synthèse). 2007. Recommandations professionnelles «Stratégie de prise en charge en cas de dénutrition protéino-énergétique chez la personne âgée» validées en avril 2007, pp. 1-4. Disponible sur https://www.has-sante.fr/portail/jcms/c_546549/ $\mathrm{fr} /$ strategie-de-prise-en-charge-en-cas-de-denutrition-proteinoenergetique-chez-la-personne-agee.

Hébrant A. 2014. La dénutrition du patient cancéreux et le danger de la supplémentation. Onco 8(6): 5.

Hébuterne X. 2012. Les acides gras n-3 sont-ils efficaces pour traiter la cachexie induite par le cancer ? La Lettre de l'HépatoGastroentérologue XV(1): 40-44.

Heckman PRA, Blokland A, Prickaerts J. 2017. From age-related cognitive decline to Alzheimer's disease: A translational overview of the potential role for phosphodiesterases. Adv Neurobiol 17: 135-168.

Henkel J, Alfine E, Saín J, et al. 2018. Soybean oil-derived polyunsaturated fatty acids enhance liver damage in NAFLD induced by dietary cholesterol. Nutrients 10: 1326 .

Herrmann FR. 1992. Serum albumin level on admission as a predictor of death, length of stay, and readmission. Arch Intern Med 152: 125-130.

Kim JM, Sung MK. 2016. The efficacy of oral nutritional intervention in malnourished cancer patients: A systemic review. Clin Nutr Res 5: 219-236.

Langen RCJ, Schols AMWJ, Kelders MCJM, van der Velden JLJ, Wouters EFM, Janssen-Heininger YMW. 2006. Muscle wasting and impaired muscle regeneration in a murine model of chronic pulmonary inflammation. Am J Respir Cell Mol Biol 35: 689-696.

Laviano A, Di Lazzaro Giraldi G, Koverech A. 2016. Does nutrition support have a role in managing cancer cachexia? Curr Opin Support Palliat Care 10: 288-292.

Lipina C, Hundal HS. 2017. Lipid modulation of skeletal muscle mass and function: Lipid regulation of skeletal muscle function. J Cachexia Sarcopenia Muscle 8: 190-201.

Marshall S. 2018. Why is the skeleton still in the hospital closet? A look at the complex aetiology of protein-energy malnutrition and its implications for the nutrition care team. J Nutr Health Aging 22: 26-29.

Martin C, Chevrot M, Passilly-Degrace P, Besnard P. 2010. Détection oro-sensorielle des lipides alimentaires : impacts sur le comportement alimentaire et la santé. Innov Agron 10: 81-93.
Milne A, Potter J, Vivanti A, Avenell A. 2009. Protein and energy supplementation in elderly people at risk from malnutrition. Cochrane Database Syst Rev 15(2): 98.

Monarque-Favard C, Garcia I, Abidi H, et al. (2002). Malnourished elderly people and lipid status. J Nutr Health Aging 6(6): 370-374.

Morais JA, Jacob KW, Chevalier S. 2018. Effects of aging and insulin resistant states on protein anabolic responses in older adults. Exp Gerontol 108: 262-268.

O'Connor JP, Manigrasso MB, Kim BD, Subramanian S. 2014. Fracture healing and lipid mediators. Bonekey Rep 2(3): 517-523.

Omran ML, Morley JE. (2000). Assessment of protein energy malnutrition in older persons, part II: Laboratory evaluation. Nutrition 16: 131-140.

Ould-Hamouda H. (2015). Thèse : impact de la qualité des protéines et des lipides du régime de renutrition sur la composition en acides gras, la réponse hépatique à l'insuline, la régulation de l'homéostasie énergétique et l'inflammation, chez les rats âgés Wistar souffrant de malnutrition. Université Paris-Sud, pp. $1-185$.

Paillaud E, Herbaud S, Caillet P, Lejonc JL, Campillo B, Bories PN. 2005. Relations between undernutrition and nosocomial infections in elderly patients. Age Ageing 34: 619-625.

Pappalardo G, Almeida A, Ravasco P. 2015. Eicosapentaenoic acid in cancer improves body composition and modulates metabolism. Nutrition 31: 549-555.

PNNS. 2010. Dénutrition, une pathologie méconnue en société d'abondance. Paris : Ministère de la santé et des sports (éditeur), pp. 1-93.

Potter J, Klipstein K, Reilly JJ, Roberts M. 1995. The nutrititional status and clinical course of acute admissions to a geriatric unit. Age Ageing 24: 131-136.

Preidis GA, Ajami NJ, Wong MC, Bessard BC, Conner ME, Petrosino JF. 2015. Composition and function of the undernourished neonatal mouse intestinal microbiome. J Nutr Biochem 26: 1050-1057.

Raynaud-Simon A, Lesourd B. 2000. Dénutrition du sujet âgé : conséquences cliniques. Presse Med 29: 2183-2190

Régl. (UE) 2016/128. 2016. Commission Européenne - RÉGLEMENT DÉLÉGUÉ (UE) 2016/128 DE LA COMMISSION - du 25 septembre 2015 - complétant le règlement (UE) n ${ }^{\circ}$ 609/2013 du Parlement européen et du Conseil en ce qui concerne les exigences spécifiques en matière de composition et d'information applicables aux denrées alimentaires destinées à des fins médicales spéciales. J Off Union Eur 14: L 25/30-L 25/43

Schneyder A. 2014. Malnutrition and nutritional supplements. Aust Prescr 37: 120-123.

Smith GI, Atherton P, Reeds ND, et al. 2011. Dietary omega-3 fatty acid supplementation increases the rate of muscle protein synthesis in older adults: A randomized controlled trial. Am J Clin Nutr 93: 402-412.

Souliac L, Bizet G, Remy S. 2010. Concilier plaisir et nutrition. Innov Agron 10: 125-136.

Tachtsis B, Camera D, Lacham-Kaplan O. 2018. Potential roles of n-3 PUFAs during skeletal muscle growth and regeneration. Nutrients 10: 309-329.

Taylor C. 2017. Importance of nutrition in preventing and treating pressure ulcers. Nurs Older People 29: 33-39.

Trabal Vilchez J. 2010. Potential usefulness of an EPA-enriched nutritional supplement on chemotherapy tolerability in cancer patients without overt malnutrition. Nutr Hosp 25(5): 736-740. 
Vaitkus JA, Celi FS. 2017. The role of adipose tissue in cancerassociated cachexia. Exp Biol Med 242: 473-481.

Whitehouse AS, Smith HJ, Drake JL, Tisdale MJ. 2001. Mechanism of attenuation of skeletal muscle protein catabolism in cancer cachexia by eicosapentaenoic acid. Cancer Res 7: 3604-3609.
Yamamoto K, Kushida M, Tsuduki T. 2018. The effect of dietary lipid on gut microbiota in a senescence-accelerated prone mouse model (SAMP8). Biogerontology 19: 367-383.

Zhang Z, Pereira S, Luo M, Matheson E. 2017. Evaluation of blood biomarkers associated with risk of malnutrition in older adults: A systematic review and meta-analysis. Nutrients 9: 829-849.

Citation de l'article : Bonhomme C. 2019. La place des lipides dans les compléments nutritionnels oraux (CNO). OCL 26: 31. 